This is the accepted version of the article:

Novio, F.; Campo, J.; Ruiz-Molina, D.. Controlling spin transition in one-dimensional coordination polymers through polymorphism. Inorganic Chemistry, (2014). 53. 16: 8742 - . 10.1021/ic5013928.

Available at: https://dx.doi.org/10.1021/ic5013928 


\section{Controlling Spin Transition in One- Dimensional Coordination Polymers through Polymorphism}

Fernando Novio ${ }^{\ddagger}$ Javier Campo $o^{\S}$ Daniel Ruiz-Molina* ${ }^{* \ddagger}$

†Centro de Investigación en Nanociencia y Nanotecnología (CIN2-CSIC) and łInstitut Catala de Nanociencia i Nanotecnología (ICN2), Campus UAB, 08193 Bellaterra, Spain $\S$ Instituto de Ciencia de Materiales de Aragón, CSIC-Universidad de Zaragoza, 50009 Zaragoza, Spain

\section{ABSTRACT}

A series of polymer 1 microcrystals with several different morphologies have been systematically synthesized by controlling experimental parameters, namely concentration of reactants, temperature, solvent nature, and the use of surfactants, and their valence tautomerism (VT) has been studied by combined electron microscopy, X-ray diffraction data, and magnetization. Our results indicate that all of them can be grouped exclusively into two different crystalline phases, or a mixture of them, that critically determine the VT process, independent of the morphology and/or dimensions of the crystals. Moreover, a difference in the critical temperature of both phases by more than $50 \mathrm{~K}$ allows us to regulate VT. These results head the use of valence tautomeric 1D polymers in devices where strict control and reproducibility of the switching behavior at different length scales and integration procedures is highly required. 


\section{INTRODUCTION}

The influence of polymorphism on spin transition coordination polymers is used to tune the critical interconversion transition temperatures by establishing novel methodologies for the morphology-selective fabrication of coordination polymer microcrystals with reproducible crystalline phases and properties.

Crystal engineering of one-dimensional coordination polymers (1D-CP) with welldefined functionalities has been shown as an efficient route to produce materials of technological relevance.(1) Of these, 1D spin transition polymers that interconvert between electronic states with different optical and magnetic properties by an external stimulus are of especial relevance.(2) Families of coordination systems that can be switched in this manner include spin crossover $(\mathrm{SCO})(3)$ and valence tautomeric (VT) complexes.(4) Several examples of spin crossovers 1D-CP have been described so far;(5) from these studies the main limitation witnessed before a real application becomes true(6) is an understanding of polymorphism effects(7) on spin transitions. Much fewer studies have been reported on VT 1D-CP systems, where a similar tendency is envisioned.(8) For this reason, further studies that allow for proper structure-function relationships are highly required.

The assembly of coordination polymers at the micro-/nanoscale (CPPs) with welldefined crystal symmetries and morphologies represents an excellent scenario for these studies. CPPs allow for a proper structure-morphology-functionality correlation(9) and, therefore, enable the rational design of new generations of coordination polymers targeting specific desired properties. For instance, the influence of the precursors,(10) nature of the metal nodes, ancillary ligand, and counterions,(11) and use of surfactants(12) or aggregation phenomena(13) on the 
final morphology/properties of CPPs have been stated. However, even though some examples of spin transition 1D-CP at the micro-/nanoscale have already been reported,(14) no detailed studies to determine morphology and crystalline phase influence on the resulting spin transition was reported.(15)

To fill this gap, we have envisioned a systematic study of several different microsized structures of the same coordination polymer. An excellent model for these studies is the one-dimensional coordination polymer 1 (see Scheme 1), bearing a cobalt metal ion and catechol ligands connected by the 4,4'-bipyridine ligand. In a previous communication polymer 1 was already obtained as amorphous nanoparticles and exhibited VT behavior with striking and unpredictable differences with respect to its crystalline counterpart.(18) Such differential behavior is not new. VT switching takes place through an intramolecular electron between the metal ion and a redox-active ligand in response to temperature modifications.(17) Although of intramolecular origin, the matrix nature has already been shown to condition such equilibria(18) as long as variations in the metal-ligand bond lengths, i.e. the density of vibrational states, are strongly dependent on the phononic relaxation.(19) Having full control of this dependence represents a challenge.

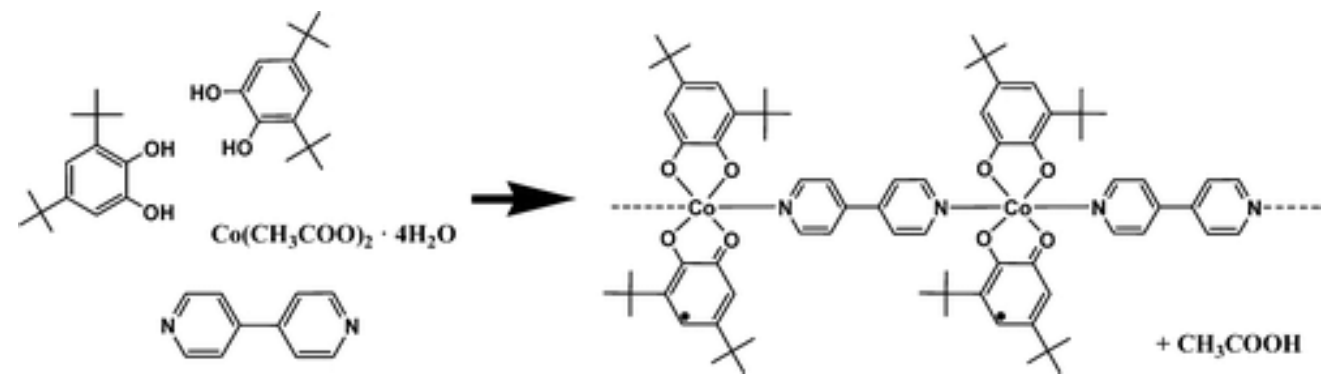

Scheme 1. Synthesis of Polymer 1 
Herein several different batches of polymer 1 have been obtained by precipitation (sometimes spontaneous) from a poor solvent. Control over the morphology and size

of the resulting precipitate is finely tuned by controlling experimental parameters, namely concentration, temperature, solvent nature, and even the use of surfactants. A detailed study by combined electron microscopy, X-ray diffraction data, and magnetization studies have allowed us for the first time to extract conclusive information about the phonon matrix influence on the VT equilibria.

\section{Experimental Section}

All operations were carried out open to air. Chemicals were obtained from different suppliers and used without further purification. The stirring time was kept constant during the different experiments to facilitate the methodological study; all of the experiments shown in this work have been done in a semistatic range (2 min of stirring at $800 \mathrm{rpm}$ ) to ensure the mixing of the reagents and the mixture was subsequently allowed to stand. Finally, the resulting CPPs were characterized by FTIR spectroscopy, X-ray diffraction, scanning electron microscopy (SEM), thermal gravimetric analysis (TGA), and magnetic measurements.

\section{Synthesis of 1: [Colll(4,4'-bipy)(3,5-dbsq)(3,5-dbcat)] ( $\alpha$ - and $\beta$-phase)}

In general, the complexes was prepared by procedures similar to those in our previous report, changing some experimental conditions.(19) In this work close to 25 different reaction conditions were performed to synthesize materials with the same chemical composition but containing different crystalline phases $(\alpha / \beta)$ depending on 
two main factors: reagent concentration and temperature. In all cases the ratio among cobalt salt, linker ligands, and catechol ligands was 1:1:2, respectively. In general, the stabilization of the $\alpha$-phase at room temperature was achieved when the concentration (referred to metallic salt) was lower than $0.01 \mathrm{M}$ : 4,4'-bipyridyl (20 mg, $0.125 \mathrm{mmol}$ ) and 3,5-di-tert-butylcatechol $(55 \mathrm{mg}, 0.25 \mathrm{mmol})$ were first dissolved in $10 \mathrm{~mL}$ of ethanol at room temperature. The addition with stirring ( $~ 800 \mathrm{rpm}$ for 2 $\mathrm{min})$ of an aqueous solution of $\mathrm{Co}(\mathrm{CH} 3 \mathrm{COO}) 2 \cdot 4 \mathrm{H} 2 \mathrm{O}(31 \mathrm{mg}, 0.125 \mathrm{mmol}$ in $2 \mathrm{~mL}$ of $\mathrm{H} 2 \mathrm{O}$ ) led to a color change to dark blue and the subsequent precipitation of the resulting product. The achievement of the $\beta$-phase at room temperature requires increasing the reagent concentration above $0.04 \mathrm{M}$ and following the same procedure. In both cases a blue crystalline precipitate of the valence tautomeric complex was washed with ethanol and isolated by filtration. Chemical analysis and FT-IR characterization corroborate the identical chemical composition. Data for the a-phase are as follows. Anal. Calcd for C38H48O4CoN2: C, 69.70; H, 7.38; N, 4.27. Found: C, 69.67; H, 7.29; N, 4.14. FT-IR (ATR mode): v 3436 (s), 2961 (s), 1608 (s), $1586(\mathrm{~s}), 1484(\mathrm{~m}), 1414(\mathrm{~m}), 806 \mathrm{~cm}-1(\mathrm{~m})$. Data for the $\beta$-phase are as follows. Anal. Calcd for C38H48O4CoN2: C, 69.70; H, 7.38; N, 4.27, Found: C, 69.81; H, 7.38; N, 4.21. FT-IR (ATR mode): v 3439 (s), 2963 (s), 1608 (s), 1587 (s), 1486 (m), $1413(\mathrm{~m}), 806 \mathrm{~cm}-1(\mathrm{~m})$.

Variations in reagent concentration, temperature, and amount of surfactants and changes in solvent result in morphological and crystalline phase modifications as detailed throughout this work. A compilation of all the reaction conditions with the corresponding morphologies and crystalline phases obtained is tabulated in the Supporting Information. 


\section{Physical Measurements}

Scanning electron microscopy (SEM) images were collected on a scanning electron microscope (HITACHI S-570) at acceleration voltages of 10-15 kV. Aluminum was used as support. Magnetic susceptibilities of samples were measured in the temperature range 5-400 K with a Quantum Design MPMS XL SQUID magnetometer operating at a magnetic field strength of $0.1 \mathrm{~T}$. Infrared (IR) spectra were performed on a FTIR Tensor 27 infrared spectrophotometer (Bruker) equipped with a Bruker Golden Gate diamond ATR (attenuated total reflection) cell. Thermal stabilities of the compounds were measured as a function of temperature and time using a simultaneous thermogravimetric analysis-differential scanning calorimetry/differential thermal analysis (TGA-DSC/DTA) system, NETZSCH-STA 449 F1 Jupiter, under nitrogen and air atmosphere and at ambient preasure. The sensitivity of the balance is $0.07 \mu \mathrm{g}$, and the furnace can operate from room temperature to $1400{ }^{\circ} \mathrm{C}$. Powder X-ray diffraction spectra were recorded at room temperature on a PANalytical X'Pert PRO MRD (high-resolution texture goniometer) working in reflection mode. The diffractometer was equipped with a Co Ka radiation source $(\lambda=1.7903 \AA)$. Ultraviolet-visible and reflectance spectra were obtained using an Agilent Cary 5000 spectrophotometer equipped with an external diffuse reflectance integrating sphere and a temperature-controlled cuvette.

\section{Results and Discussion}

In a typical reaction, an aqueous solution of $\mathrm{Co}(\mathrm{CH} 3 \mathrm{COO}) 2 \cdot 4 \mathrm{H} 2 \mathrm{O}$ is added to an ethanol solution containing 1 equiv of 4,4'-bipyridyl and 2 equiv of 3,5-di-tertbutylcatechol at $25{ }^{\circ} \mathrm{C}$, resulting in the production of a blue crystalline precipitate 
within a few minutes, identified after filtration as polymer 1 . The powder diffraction pattern of a sample obtained by using a $0.01 \mathrm{M}$ concentration of $\mathrm{Co}(\mathrm{CH} 3 \mathrm{COO}) 2 \cdot 4 \mathrm{H} 2 \mathrm{O}$ (while keeping constant the molar ratios of the two ligands) nicely fits with that simulated from the single-crystal X-ray data (monoclinic space group C2/c) previously reported (see S2 in the Supporting Information),(16) and this sample is denoted the a-phase from now on. The peaks are strong and narrow, indicating the good crystallinity of the as-prepared sample, with no clear evidence for additional peaks arising from any other phase or impurity (see Figure 1a). The structure lacks solvent molecules within the crystalline network, a fact that was confirmed by chemical analysis and TGA measurements (see S3 in the Supporting Information). A gradual increase of the reagent concentration, while the rest of the experimental parameters are maintained constant, reveals the appearance of new peaks in the X-ray powder diffractogram and the gradual decrease of those already existing (Figure 1b). The production of a new stabilized phase is observed when the cobalt salt concentration is increased to $0.04 \mathrm{M}$ (while keeping constant the molar ratios of the two ligands). As a representative example of this dependence, the X-ray powder diffraction data of samples obtained at three different concentrations $(0.01$, 0.02 , and $0.04 \mathrm{M}$ ) are shown in Figure $1 \mathrm{~b}$. Unfortunately, and in spite of the several efforts made by both direct X-ray single-crystal analysis and X-ray powder diffraction data simulation, the structure of the new phase, from now on denoted the $\beta$-phase, remains elusive. 


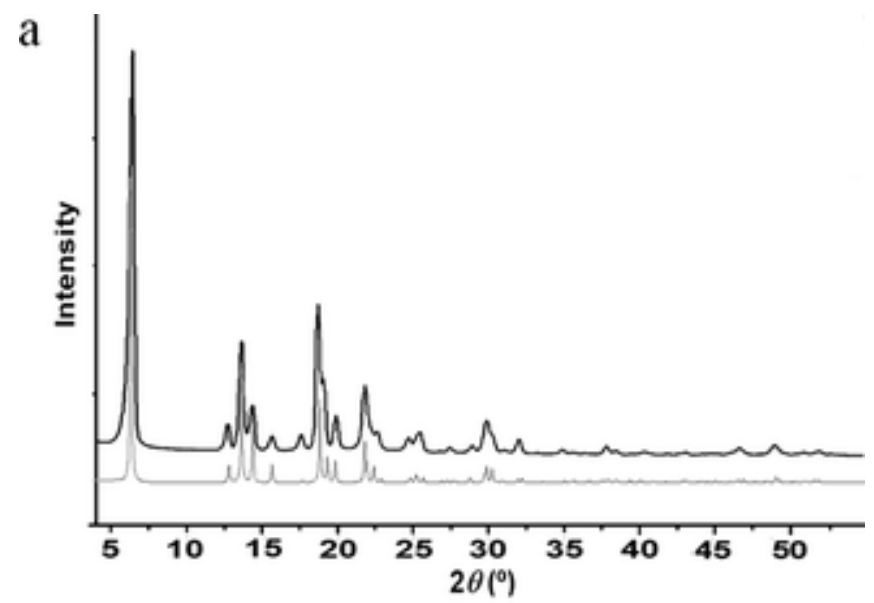

b

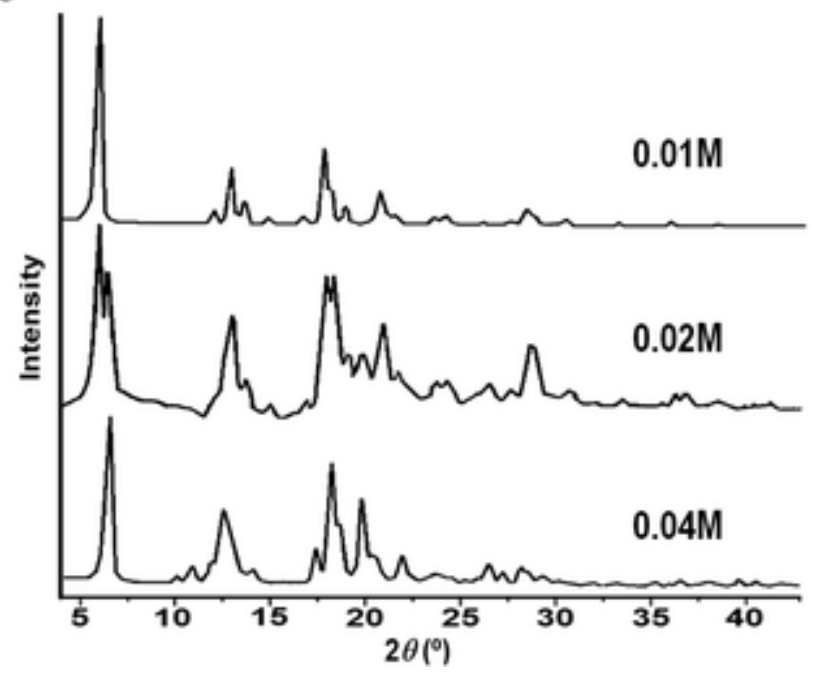

Figure 1. (a) XRD pattern for $\alpha$-phase (black line) and the corresponding simulated spectrum (gray line). (b) XRD spectra for $\alpha$-phase $(0.01 \mathrm{M}),(\alpha+\beta)$-phase $(0.02 \mathrm{M})$, and $\beta$-phase $(0.04 \mathrm{M})$ obtained at different reagent concentrations.

$\mathrm{C}, \mathrm{H}$, and $\mathrm{N}$ chemical analysis, FT-IR spectra, and magnetic measurements (vide infra) of the new phase are in excellent agreement with those obtained for the $\alpha$ phase. Thermal analysis of both phases is also in good agreement with a major weight loss (ca. 55\%) at $250{ }^{\circ} \mathrm{C}$, corresponding to decomposition (see S3 in the Supporting Information). The performance of different thermal cycles from room temperature to $175{ }^{\circ} \mathrm{C}$ (below the decomposition temperature) corroborated the 
robustness of the material with no conversion between both crystalline phases, as confirmed by X-ray powder diffraction data.

SEM images reveal remarkable morphology differences for both crystalline phases (Figure 2). The $\beta$-phase consists of rodlike submicrocrystals with a long aspect ratio, several micrometers in length and a few hundred nanometers in width. In contrast, the $\alpha$-phase exhibits a spongelike shape several micrometers in diameter composed by superstructures of thin crystallites (Figure $2 a, b$ ). It is worth mentioning that intermediate concentrations lead to the formation of related spongelike superstructures. To get more insight into these morphological divergences, the crystal growth of polymer 1 was monitored through time by combined SEM and X-ray diffraction experiments (see Figure 2c,d). Crystal growth involves two main steps: an initial nucleation and a subsequent crystal growth stage. In our case, higher concentrations induce an increase of the nucleation rate, i.e., more seeds are formed in a shorter time, which afterward grow slowly, avoiding splitting growth processes. Accordingly, rodlike microcrystals with a high anisotropy are formed, in agreement with the chemical anisotropy of 1 (see Figure 2c). A concentration decrease results in the formation of fewer seeds with a subsequent faster radial growth and formation of the spongelike crystals (see Figure $2 \mathrm{~d}$ ). 


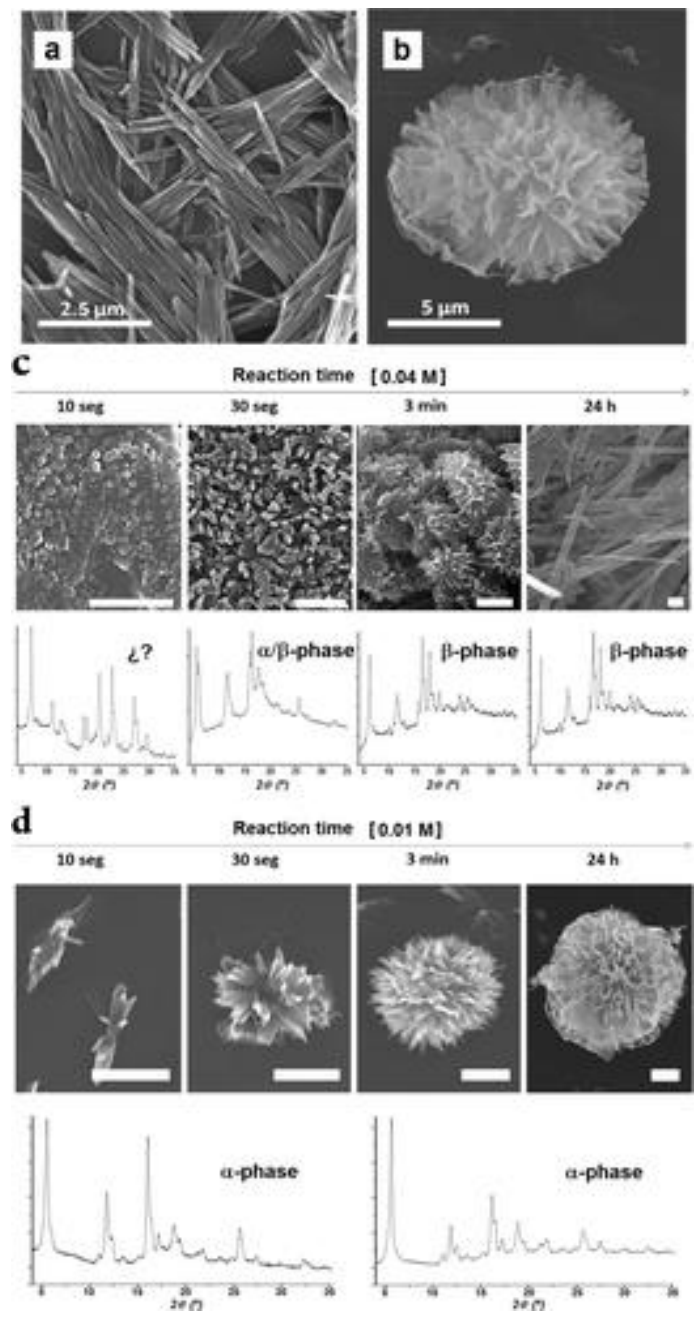

Figure 2. (a) SEM image of polymer 1 crystals obtained using salt concentrations of 0.04 M. (b) SEM images of polymer 1 crystals obtained at the lowest salt concentration of $0.01 \mathrm{M}$. Time evolution of both crystalline structures at room temperature: (c) at $0.04 \mathrm{M}$ ( $\beta$-phase); (d) $0.01 \mathrm{M}$ ( $\alpha$-phase). Scale bar: $1 \mu \mathrm{m}$.

\section{Temperature Effect}

To study the effect of temperature on the crystal growth of polymer 1, new experiments at two additional temperatures $\left(0\right.$ and $\left.70{ }^{\circ} \mathrm{C}\right)$ and three different salt concentrations $(0.01,0.02$, and $0.04 \mathrm{M})$, with the rest of the parameters maintained constant, were done. A detailed recompilation of all the morphologies obtained is 
shown in Figure 3. A temperature decrease from 25 to $0{ }^{\circ} \mathrm{C}$ has no influence on the final morphologies except at the intermediate concentration of $0.02 \mathrm{M}$, where the morphology changes from spongelike to fibers with a very long aspect ratio. The most significant changes are found upon increasing the temperature from 25 to 70 ${ }^{\circ} \mathrm{C}$. In this case thin plates a few micrometers in width and a hundred nanometers thick (organized as flowerlike superstructures at a concentration of $0.02 \mathrm{M}$ ) are obtained. It is worth mentioning that significant decomposition processes are observed before crystallization of 1 at the lowest concentration of $0.01 \mathrm{M}$. 


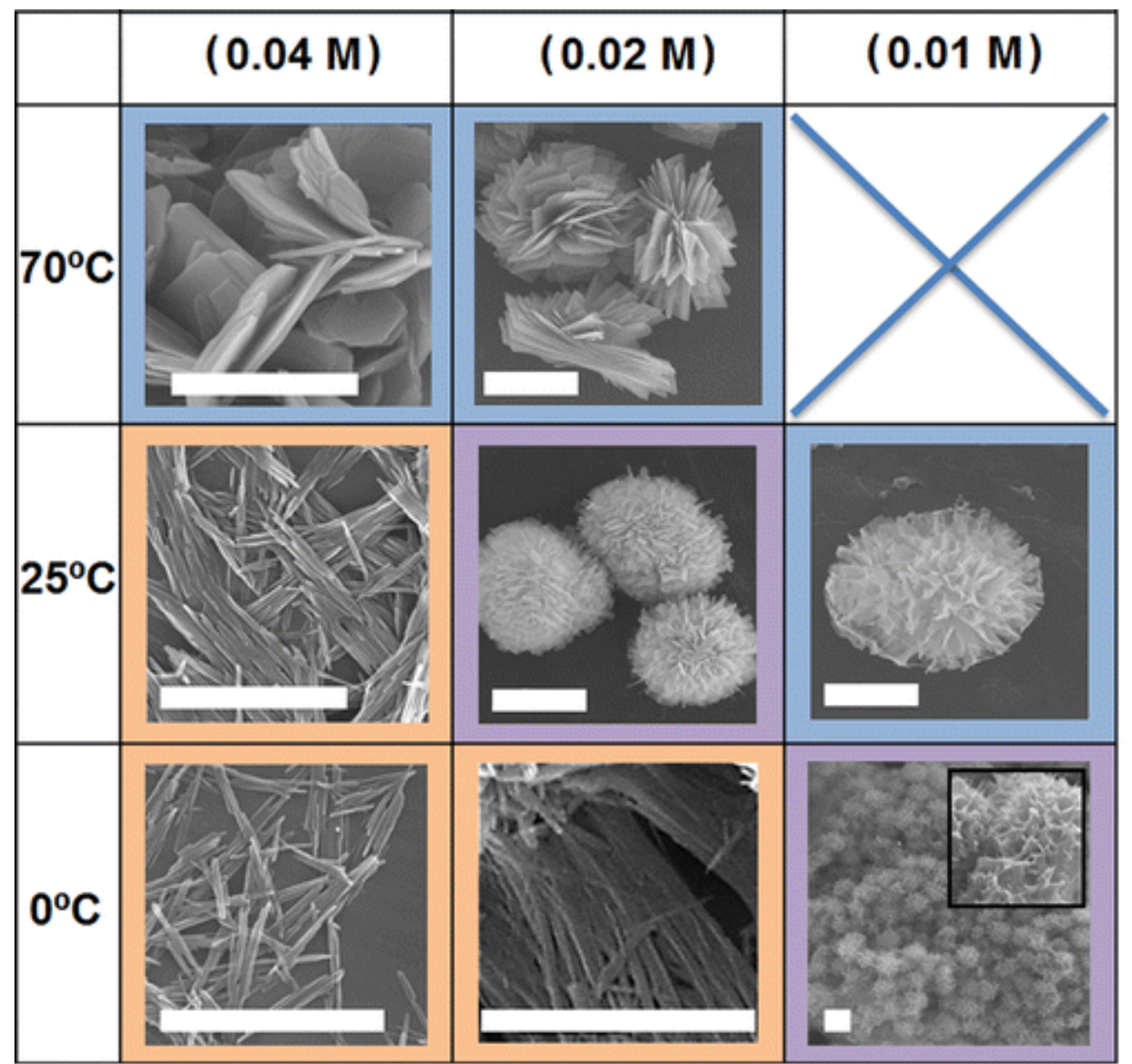

Figure 3. SEM images of polymer 1 microcrystals obtained at different reagent concentrations and temperatures, as indicated in the figure. For the lowest concentration of $0.01 \mathrm{M}$ at $70{ }^{\circ} \mathrm{C}$, significant decomposition processes are observed before crystallization of 1 . The different crystalline phases obtained are indicated with an arbitrary color code: $\alpha$-phase (blue), $\alpha+\beta$-phase (violet), $\beta$-phase (orange). Scale bar: $5 \mu \mathrm{m}$.

As far as the crystal phase is concerned, we only obtain one of the two phases, either the $\alpha$-phase or $\beta$-phase, or a combination of both. No appearance of peaks associated with new phases was detected. At $0{ }^{\circ} \mathrm{C}$ the $\alpha$-phase is predominant, while at $70{ }^{\circ} \mathrm{C}$ the predominant phase is the $\beta$-phase; at an intermediate temperature 
of $25^{\circ} \mathrm{C}$ the phase is strongly dependent on the reagent concentrations (see Figure 3).

\section{Use of Surfactants}

The surfactants used (in all cases below 5\%) were the polymeric polyvinylpyrrolidone (PVP) and the anionic surfactant sodium dodecylbenzenesulfonate (SDS). As can be seen in Figure 4, the use of PVP clearly favors the formation of spherical flowerlike superstructures composed of several tiny plates (the higher the initial cobalt salt concentration, the more plates contain the crystal). This morphology does not represent a significant modification from that obtained in the absence of surfactant at low reagent concentrations. More striking differences are observed at higher concentrations, where submicrocrystals with a long aspect ratio are obtained in the absence of PVP. Moreover, the higher the PVP ratio used, the smaller the dimensions of the superstructures obtained. A plausible explanation for these observations can be found upon considering the crystal formation mechanism in the presence of PVP,(20) which consists of two main steps. In the first step, the polymerization reaction starts and the coordination polymer begins to aggregate at close range, primarily as nanocrystal seeds within the polymeric backbone. The second step consists of the nanostructure growth within the polymeric holes. In this last step the concentration of PVP is critical, since the polymer hole size, which determines the size of the resulting nanostructure, decreases as the polymer concentration is increased. 


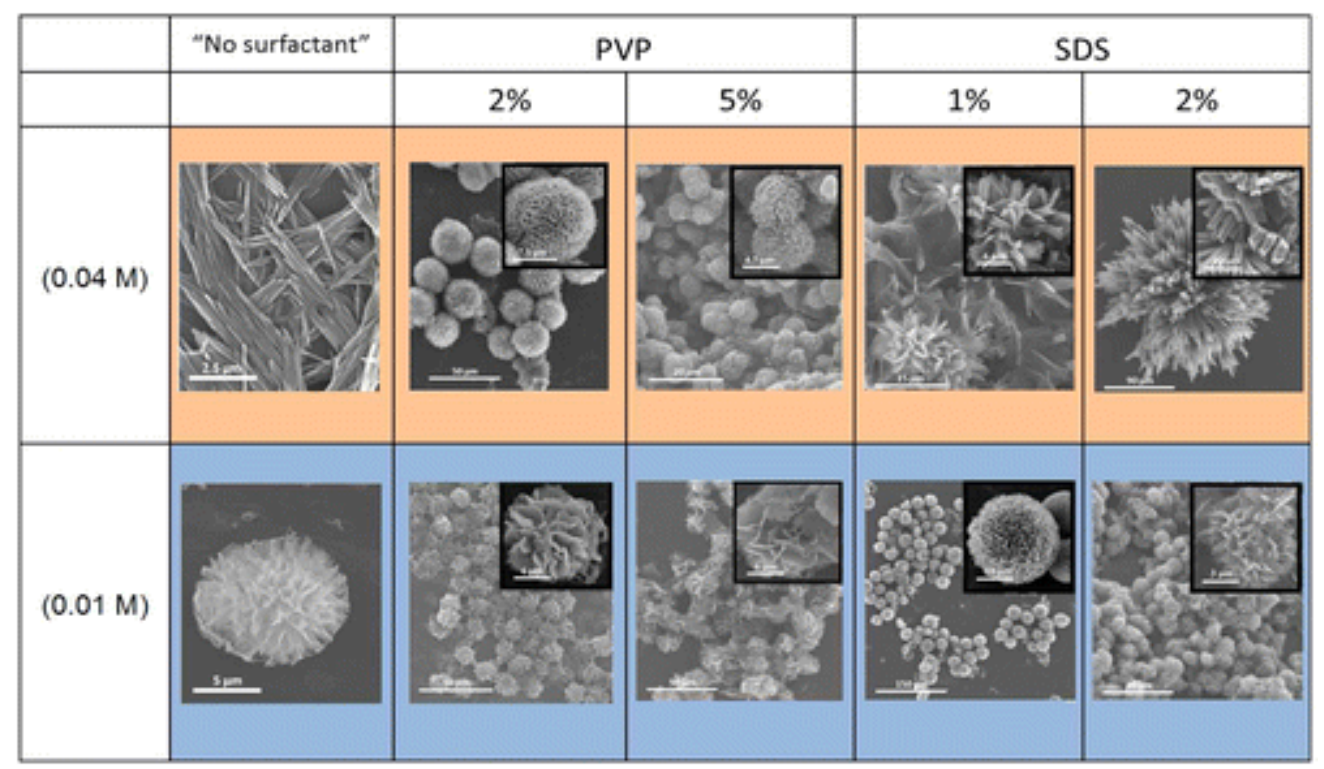

Figure 4. SEM images of micro-/nanostructures of 1 obtained at 0.01 and $0.04 \mathrm{M}$ concentrations of the metal salt and using different surfactant (PVP or SDS) concentration. Color code: $\alpha$-phase (blue), $\beta$-phase (orange).

Microcrystals obtained by using SDS (both 1 and 2\%) at the lowest cobalt salt concentration exhibit once more spherical flowerlike superstructures, similar to that obtained without or with PVP as surfactant. More remarkable morphology variations are found upon increasing the metal salt concentration, where crystalline superstructures made of several rectangular rods are found. Most likely, an increase of the SDS ratio induces less aggregation of the nanocrystal seeds, allowing for an increase in the length and width of the primary crystals.(21)

Finally, the use of surfactants does not modify the crystalline phase of the resulting material, it being dictated by the initial concentration of the cobalt salt. Moreover, it was confirmed that the surfactants do not take part in the final material composition. 


\section{Solvent Effects}

In addition to EtOH, DMF and THF were also used, due to the good solubility of the ligands and their miscibility with water. SEM images of the aforementioned structures are shown in Figure 5. Interestingly, all the microcrystals obtained in THF consist of superstructures hierarchically formed by very thin needles that range from urchinlike to unstructured samples, depending on the concentration or temperature used. A more broad range of morphologies is found by using DMF. Depending on both the initial cobalt salt concentration and/or the temperature, several different morphologies ranging from urchinlike to plates or flowerlike supersturctures are obtained. As far as the crystal phase is concerned, all the microcrystals obtained in THF yield the $\beta$-phase independent of the concentration or temperature used. In contrast, the crystal phase of the samples obtained in DMF changes depending on the initial metal salt concentration and/or temperature, in a manner similar to that observed for the structures obtained in ethanol. 


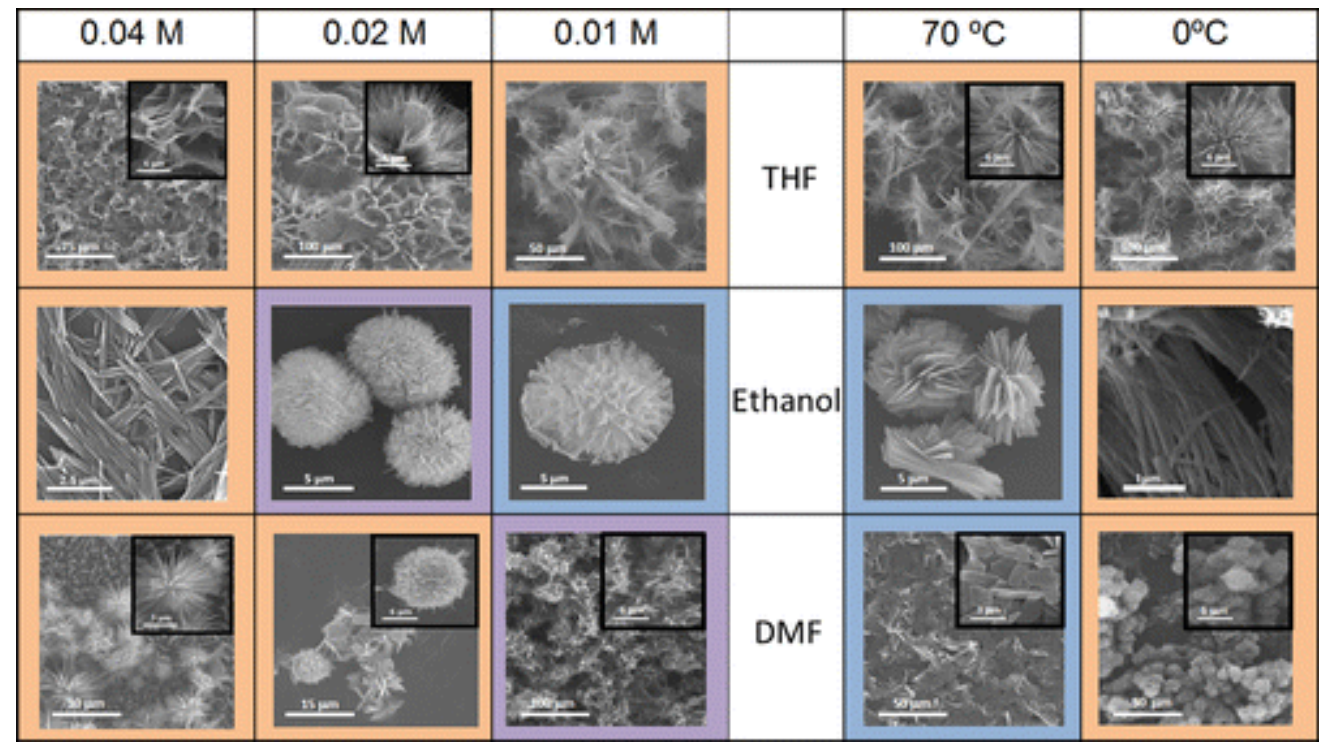

Figure 5. SEM images of micro-/nanostructures of 1 obtained in different solvents at $0.01,0.02$, and $0.04 \mathrm{M}$ concentrations (right) and at different temperatures (left). Color code: $\alpha$-phase (blue), $\alpha+\beta$-phase (violet), $\beta$-phase (orange).

\section{Magnetic Measurements}

Magnetic susceptibility data were measured for all the different morphologies in the 5-380 K temperature range. All of them can be grouped into two different magnetic behaviors (or a combination of them), depending on the crystalline phase and independent of the morphology. Representative examples of magnetization curves found for the $\alpha$-phase and $\beta$-phase and their mixtures are shown in Figure $6 a$. 

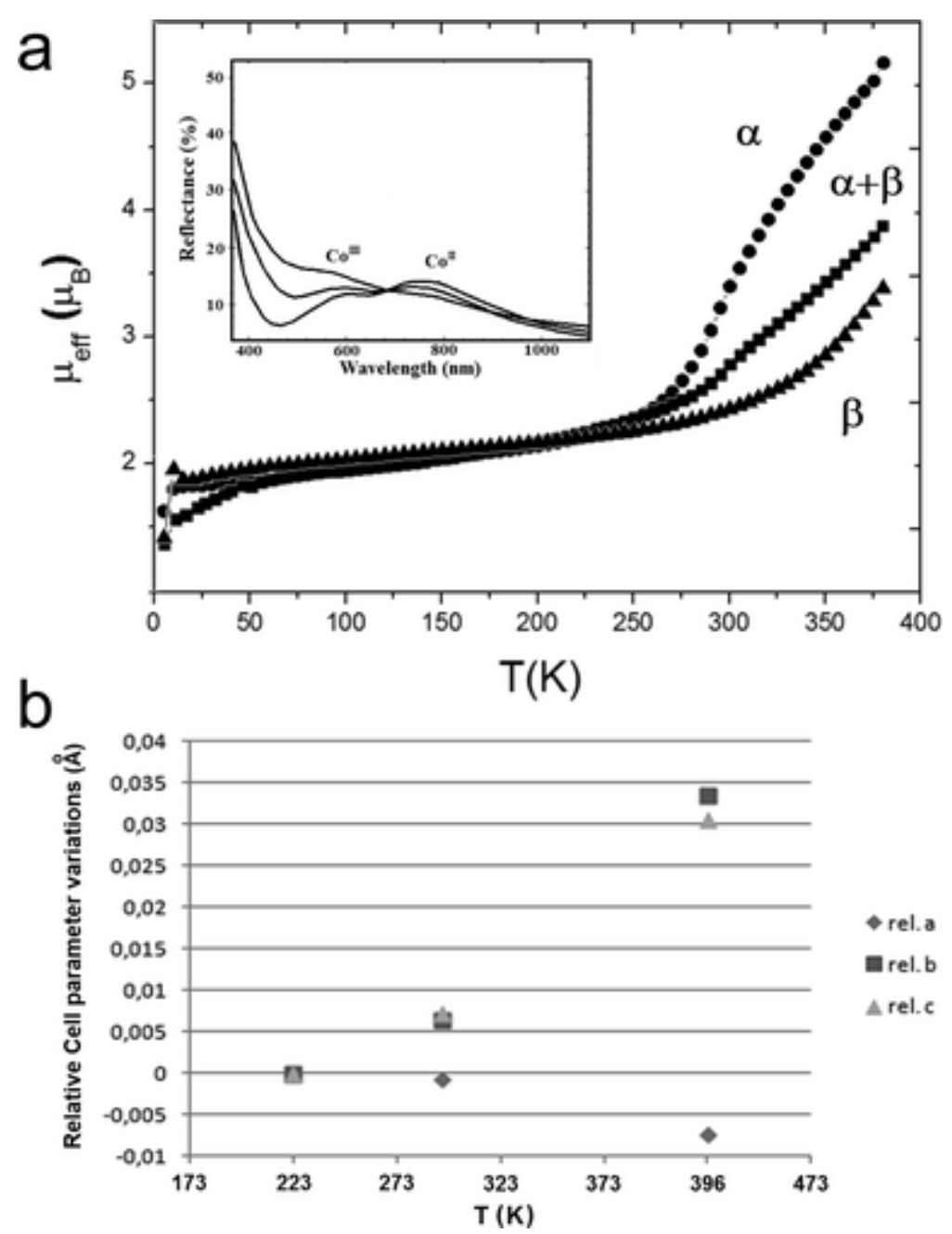

Figure 6. (a) Magnetic measurements for the different crystalline phases as a function of temperature. The inset gives the UV-vis spectra of the a-phase between 253 and 333 K. (b) Structural changes in the unit cell (C2/c) parameters as a result of increased temperature from $-50^{\circ} \mathrm{C}(223 \mathrm{~K})$ to $150^{\circ} \mathrm{C}(423 \mathrm{~K})$.

In the low-temperature range of $5-250 \mathrm{~K}$ only the Is-[CollI(4,4'-bipy)(3,5-dbsq)(3,5dbcat)] ( $S=1 / 2)$ tautomer is observed in all cases. As the temperature is increased, ligand-to-metal intramolecular electron transfer is activated as one of the ligands evolves from the catecholate form $(3,5$-dbcat) to the semiquinone radical ligand $(3,5$ dbsq). Thus, at a given temperature the Is-Colll tautomer converts into the hs-Coll 
form, accompanied by an increase in magnetic moment. In the $\alpha$-phase the transition starts at $275 \mathrm{~K}$ with a maximum value of $\mu$ eff $=5.3 \mu \mathrm{B}$ reached at $380 \mathrm{~K}$, corroborating almost a complete conversion to the hs-Coll isomer. However, in the $\beta$-phase the magnetic change does not takes place until $325 \mathrm{~K}$ and the conversion is incomplete at $380 \mathrm{~K}$, involving a shift of the critical interconversion temperature (Tc) by more than $50 \mathrm{~K}$. Changes in the crystalline phase for the same complex induce a tuning in the Tc value. As expected, an intermediate situation is obtained for mixtures of both phases. These crystalline materials endure several thermal cycles in the solid state from 223 to $450 \mathrm{~K}$ without any significant change in the magnetization behavior, a fact that was confirmed by elemental analysis. Above 450 $\mathrm{K}$ the material starts to degrade, yielding a complex mixture of products that is difficult to characterize. The VT equilibrium was also confirmed by UV-vis spectrometry at different temperatures, showing the prevalence of each isomer on the basis of the different typical bands corresponding to both tautomers at 580 and $720 \mathrm{~nm}$ for Is-Colll and hs-Coll, respectively. An increase in the temperature enhances the $720 \mathrm{~nm}$ band and decreases the $580 \mathrm{~nm}$ band, corroborating the assignation of signals (see inset spectra in Figure 6a).

Finally, we also monitored changes in the unit cell (C2/c) parameters upon increasing the temperature from 223 to $423 \mathrm{~K}$, where the VT has been shown to take place (calculation details are presented in S4 in the Supporting Information). As can be seen in Figure 6b, an increase of the Co-N bond length by ca. $0.2 \AA$ ( $0.36 \AA$ if an increase of ionic radius from Is-Colll to hs-Coll is included) is observed, involving a significant expansion along the $b$ and $c$ axes:(22) i.e., the polymeric chain propagation vector. These changes imply an increase of the total cell volume of 218 Å3 along the temperature range studied, though the most abrupt volume variation 
(171 Å3) is observed between room temperature and $423 \mathrm{~K}$. This is the range where the largest changes in the effective magnetic moment are found. These structural changes are reversible over several cycles, as previously observed by variabletemperature magnetization data.

\section{CONCLUSIONS}

By controlling experimental reaction parameters, several microparticles of polymer $\mathbf{1}$ have been obtained and studied by combined X-ray diffraction and SEM studies (a detailed list of all the different reactions and morphologies obtained is given in S1 in the Supporting Information). Interestingly, all of them can be exclusively grouped into two crystalline phases or a mixture of them. Moreover, the VT transition is conditioned by the crystal symmetry of the phase independent of the crystal morphology and/or dimensions. The use of low reactant concentrations at room temperature (or below) favors the $\alpha$-phase, where the transition starts at $275 \mathrm{~K}$ with a maximum value of $\mu_{\text {eff }}=5.3 \mu_{\mathrm{B}}$ reached at $380 \mathrm{~K}$. A 4-fold increase of the concentration above room temperature ensures the formation of the $\boldsymbol{\beta}$-phase, which exhibits a $T_{\mathrm{c}}$ shift by more than $50 \mathrm{~K}$. Therefore, the possibility of controlling the fabrication of microparticles with specific crystal symmetry allow us for the first time to design VT microparticles with reproducible switching and controlled VT temperatures, a major problem in the design of future molecular devices based on these materials. 


\section{SUPPORTING INFORMATION}

Text, figures, and tables giving a summary of the experimental conditions and the corresponding morphologies and crystalline phase of the [CollI(4,4'-bipy)(3,5dbsq)(3,5-dbcat)] samples obtained, X-ray data for 1 , thermogravimetric analysis of the $\alpha$ - and $\beta$-phase of polymer 1 , and cell parameters extracted from XRD data at different temperatures.

\section{AUTHOR CONTRIBUTIONS}

F.N. synthesized and characterized all the different samples. J.C. was in charge of the magnetic characterization, and D.R.-M. coordinated the work and contributed with fruitful discussions. The manuscript was written through contributions of all authors. All authors have given approval to the final version of the manuscript.

\section{FUNDING INFORMATION}

This work was supported by projects MAT-2011-27233-C02-02 and MAT201238318-C03-02 from the Spanish Government and by FEDER funds.

The authors declare no competing financial interest.

\section{ACKNOWLEDGMENT}

F.N. thanks the Ministerio de Ciencia e Innovación (MICINN) for a postdoctoral JdC fellowship. We thank Dr. Norberto Masciocchi of the Department of Chemical and Environmental Sciences at the University of Uninsubria (Varese, Italy) for help with the XRD spectra discussions, and COST ECOSTBio. 


\section{REFERENCES}

1 (a) Leong, W. L.; Vittal, J. J. Chem. Rev. 2011, 111, 688- 764 (b) Chen, Ch.-T.; Suslick, K. S. Coord. Chem. Rev. 1993, 128, 293- 322

2(a) Muñoz, M. C.; Real, J. A. In Spin-Crossover Materials; Halcrow, M. A., Ed.; Wiley: Chichester, U.K., 2013; pp 121- 146. (b) Muñoz, M. C.; Real, J. A. Coord. Chem. Rev. 2011, 255, 2068- 2093

3 Spin-Crossover Materials-Properties and Applications; Halcrow, M. A., Ed.; Wiley: Chichester, U.K., 2013.[Crossref], Google Scholar

4Tezgerevska, T.; Alley, K. G.; Boskovic, C. Coord. Chem. Rev. 2014, 268, 23- 40 (b) Evangelio, E.; Ruiz-Molina, D. Eur. J. Inorg. Chem. 2005, 2957- 2971

5(a) Haryono, M.; Heinemann, F. W.; Petukhov, K.; Gieb, K.; Müller, P.; Grohmann, A. Eur. J. Inorg. Chem. 2009, 2136- 2143 (b) Galet, A.; Gaspar, A. B.; Munoz, M. C.; Levchenko, G.; Real, J. A. Inorg. Chem. 2006, 45, 9670- 9679 (c) Faulmann, C.; Szilágyi, P. Á.; Chahine, K. J.; Valade, L. New J. Chem. 2009, 33, 1268- 1276 (d) Matouzenko, G. S.; Jeanneau, E.; Verat, A. Yu.; Bousseksou, A. Dalton Trans. 2011, 40, 9608- 9618 (e) Šalitroš, I.; Fuhr, O.; Eichhöfer, A.; Kruk, R.; Pavlik, J.; Dlháň, L.; Boča, R.; Ruben, M. Dalton Trans. 2012, 41, 5163- 5171 (f) Nowak, R.; Bauer, W.; Ossiander, T.; Weber, B. Eur. J. Inorg. Chem. 2013, 975- 983 (g) Bushuev, B.; Daletsky, V. A.; Pishchur, D. P.; Gatilov, Y. V.; Korolkov, I. V.; Nikolaenkovac, E. B.; Krivopalov, V. P. Dalton Trans. 2014, 43, 39063910

6Halcrow, M. A. Chem. Soc. Rev. 2011, 40, 4119- 4142

7(a) Moulton, B.; Zaworotko, M. J. Chem. Rev. 2001, 101, 1629- 1658 (b) Guionneau, Ph. Dalton Trans. 2014, 43, 382- 393 (c) Tao, J.; Wei, R.-J.; Huang, R.-B.; Zheng, L.-S. Chem. Soc. Rev. 2012, 41, 703- 737

8(a) Calzolari, A.; Chen, Y.; Lewis, G. F.; Dougherty, D. B.; Shultz, D.; Nardelli, M. B. J. Phys. Chem. B 2012, 116, 13141- 13148 (b) Affronte, M.; Beni, A.; Dei, A.; Sorace, L. Dalton Trans. 2007, 5253- 5259 (c) Xiao, D.-R.; Wang, E.-B.; An, H.-Y.; Li, Y.-G.; Xu, L. Cryst. Growth Des. 2007, 3, 506- 512 (d) Shaikh, N.; Goswami, S.; Panja, A.; Sun, H.-L.; Pan, F.; Gao, S.; Banerjee, P. Inorg. Chem. 2005, 44, 9714- 9722 (e) Bodnar, S. H.; Caneschi, A.; Dei, A.; Shultz, D. A.; Sorace, L. Chem. Commun. 2001, 2150- 2151 (f) Attiat, A. S.; Pierpont, C. G. Inorg. Chem. 1995, 34, 1172- 1179 (g) Jung, O.-S.; Pierpont, C. G. J. Am. Chem. Soc. 1994, 116, 2229- 2230

9Spokoyny, A. M.; Kim, D.; Sumrein, A.; Mirkin, C. A. Chem. Soc. Rev. 2009, 38, 12181228

10Fan, Y.; Liu, R.; Du, W.; Lu, Q.; Pangab, H.; Gao, F. J. Mater. Chem. 2012, 22, 12609 12617

11(a) Jeon, Y.-M.; Heo, J.; Mirkin, C. A. J. Am. Chem. Soc. 2007, 129, 7480- 7481 (b) Lu, W.; Chui, S. S.-Y.; Ng, K.-M.; Che, C.-M. Angew. Chem., Int. Ed. 2008, 47, 4568- 4572 (c) Liu, Y.; Boey, F.; Lao, L. L.; Zhang, H.; Liu, X.; Zhang, Q. Chem. Asian. J. 2011, 6, 10041006

12(a) Yuan, G.; Zhu, C.; Liu, Y.; Cui, Y. Chem. Commun. 2011, 47, 3180- 3182 (b) Shi, N.; Xie, L.; Sun, H.; Duan, J.; Yin, G.; Xub, Z.; Huang, W. Chem. Commun. 2011, 47, 50555057 (c) Yuana, Y.; Wanga, W.; Qiua, L.; Penga, F.; Jianga, X.; Xiea, A.; Shena, Y.; Tianb, 
X.; Zhang, L. Mater. Chem. Phys. 2011, 131, 358-361(d) Cho, W.; Lee, H. J.; Oh, M. J. Am. Chem. Soc. 2008, 130, 16943- 16946

13(a) Chakraborty, S.; Kravitz, J. Y.; Thulstrup, P. W.; Hemmingsen, L.; DeGrado, W. F.; Pecoraro, V. L. Angew. Chem., Int. Ed. 2008, 47, 2049- 2053 (b) Zhao, J.; Guo, Y.; Guo, H.; Chai, Y.; Li, Y.; Liu, Y.; Liu, C. Inorg. Chem. Commun. 2012, 18, 21- 24

14(a) Forestier, T.; Mornet, S.; Daro, N.; Nishihara, T.; Mouri, S.-I.; Tanaka, K.; Fouche, O.; Freysz, E.; Letard, J.-F. Chem. Commun. 2008, 36, 4327- 4329 (b) Coronado, E.; GalánMascarós, J. R.; Monrabal-Capilla, M.; García-Martínez, J.; Pardo-lbáñez, P. Adv. Mater. 2007, 19, 1359- 1361 (c) Kuroiwa, K.; Kikuchi, H.; Kimizuka, N. Chem. Commun. 2010, 46, 1229- 1231 (d) Seredyuk, M.; Gaspar, A. B.; Ksenofontov, V.; Galyametdinov, Y.; Verdaguer, M.; Villain, F.; Gütlich, P. Inorg. Chem. 2008, 47, 10232- 10245 (e) Roubeau, O. Chem. Eur. J. 2012, 18, 15230- 15244 (f) Dîrtu, M. M.; Neuhausen, C.; Naik, A. D.; Rotaru, A.; Spinu, L.; Garcia, Y. Inorg. Chem. 2010, 49, 5723- 5736 (g) Neville, S. M.; Leita, B. A.; Halder, G. J.; Kepert, C. J.; Moubaraki, B.; Létard, J.-F.; Murray, K. S. Chem. Eur. J. 2008, 14, 10123- 10133 (h) Murray, K. S. Eur. J. Inorg. Chem. 2008, 3101- 3121 (i) Ross, T. M.; Moubaraki, B.; Batten, S. R.; Murray, K. S. Dalton Trans. 2012, 41, 2571 (j) Scott, H. S.; Ross, T. M.; Chilton, N. F.; Gass, I. A.; Moubaraki, B.; Chastanet, G.; Paradis, N.; Lètard, J.F.; Vignesh, K. R.; Rajaraman, G.; Batten, S. R.; Murray, K. S. Dalton Trans. 2013, 42, 16494- 16509 (k) Ross, T. M.; Moubaraki, B.; Turner, D. R.; Halder, G. J.; Chastanet, G.; Neville, S. M.; Cashion, J. D.; Létard, J.-F.; Batten, S. R.; Murray, K. S. Eur. J. Inorg. Chem. 2011, 1395- 1417

15Bousseksou, A.; Molnár, G.; Salmon, L.; Nicolazzi, W. Chem. Soc. Rev. 2011, 40, 33133335

16Imaz, I.; Maspoch, D.; Rodriguez-Blanco, C.; Perez-Falcon, J. M.; Campo, J.; RuizMolina, D. Angew. Chem., Int. Ed. 2008, 47, 1857- 1860

17(a) Dei, A.; Gatteschi, D. Angew. Chem., Int. Ed. 2011, 50, 11852- 11858 (b) Sato, O.; Cui, A.; Matsuda, R.; Tao, J.; Hayami, S. Acc. Chem. Res. 2007, 40, 361 (c) Hendrickson, D. N.; Pierpont, C. G. Top. Curr. Chem. 2004, 234, 63- 95 (d) Pierpont, C. G. Coord. Chem. Rev. 2001, 216, 99- 125 (e) Shultz, D. A. In Magnetism: Molecules to Materials; Miller, J. S., Drillon, M., Eds.; Wiley-VCH: Weinheim, Germany, 2001; Vol. II, pp 81-306. (f) GWtlich, P.; Garcia, Y.; Goodwin, H. A. Chem. Soc. Rev. 2000, 29, 419- 427 (g) Gütlich, Ph.; Dei, A. Angew. Chem., Int. Ed. Engl. 1997, 36, 2734- 2736

18Evangelio, E.; Ruiz-Molina, D. C. R. Chim. 2008, 11, 1137- 1154

19Evangelio, E.; Rodriguez-Blanco, C.; Coppel, Y.; Hendrickson, D. N.; Sutter, J. P.; Campo, J.; Ruiz-Molina, D. Solid State Sci. 2009, 11, 793-800

20(a) Sun, Y.; Xia, Y. Science 2002, 298, 2176- 2179 (b) Lu, G.; Li, S.; Guo, Z.; Farha, O. K.; Hauser, B. G.; Qi, X.; Wang, Y.; Wang, X.; Han, S.; Liu, X.; DuChene, J. S.; Zhang, H.; Zhang, Q.; Chen, X.; Ma, J.; Chye, S.; Loo, J.; Wei, W. D.; Yang, Y.; Hupp, J. T.; Huo, F. Nat. Chem. 2012, 4, 310- 316 (c) Hu, L.; Zhang, P.; Chen, Q.; Zhong, H.; Hu, X.; Zheng, X.; Wang, Y.; Yan, N. Cryst. Growth Des. 2012, 12, 2257- 2264 (d) Yin, Y.-Y.; Ma, J.-G.; Niu, Z.; Cao, X.-C.; Shi, W.; Cheng, P. Inorg. Chem. 2012, 51, 4784- 4790 (e) Goel, A.; Rani, N. Open J. Inorg. Chem. 2012, 2, 67-73

21(a) Whetten, R. L.; Gelbart, W. M. J. Phys. Chem. 1994, 98, 3544- 3549 (b) Yuan, Y.; Wang, W.; Qiu, L.; Peng, F.; Jiang, X.; Xie, A.; Shen, Y.; Tian, X.; Zhang, L. Mater. Chem. Phys. 2011, 131, 358- 361

22Soltani, N.; Saion, E.; Erfani, M.; Rezaee, K.; Bahmanrokh, G.; Drummen, G. P. C.; Bahrami, A.; Hussein, M. Z. Int. J. Mol. Sci. 2012, 13, 12412- 12427 